\title{
Evaluación de densidades de siembra sobre el rendimiento de Ají Páprika (Capsicum annuum L.) var. Bella
}

\section{Evaluation of planting densities on the yield of Ají Páprika (Capsicum annuum L.) var. Bella}

\author{
Daniel Cruz Nieto ${ }^{1}$, José Legua Cárdenas ${ }^{1}$, Yasmin Vélez Chang ${ }^{1}$, Félix Caro Soto ${ }^{1}$ Abel Eduardo, Cruz Nieto
}

\begin{abstract}
RESUMEN
Objetivo: Evaluar la densidades de siembra respecto al rendimiento del ají páprika (Capsicum annuum L.), en la variedad Bella, para determinar que distanciamiento obtuvo mayor rendimiento y calidad en el Sector La Florida - Provincia de Barranca. Material y Métodos: Se utilizó el diseño de bloques completamente al azar, conformado con cuatro tratamientos y cuatros bloques y para la operación de los datos el análisis de varianza y la prueba de Duncan. Se evaluaron desde el trasplante hasta post cosecha, en los distanciamientos $(T)$ de $T_{1}=20 \mathrm{~cm}, T_{2}=25 \mathrm{~cm}, T_{3}=30 \mathrm{~cm}$ y $T_{4}=35 \mathrm{~cm}$ a $90 \mathrm{~cm}$ entre surco. Resultados: El tratamiento $T_{4}=35 \mathrm{~cm}$. (mayor distanciamiento), sobresalió en los siguientes parámetros como: 97,25\% porcentaje de prendimiento, 75,25 cm. altura de planta, 15 frutos por planta; 33 flores por planta; 10 frutos maduros por planta; 30,51 g. de frutos fresco; $6,99 \mathrm{~g}$ de fruto seco por planta; $14,76 \mathrm{~cm}$. de longitud de fruto; $3,46 \mathrm{~cm}$ de diámetro, 0,50 cm. Pese a que el $T_{4}$ mostró buenas características; el $T_{1}=20 \mathrm{~cm}$. (menor distanciamiento), también se obtuvo buenos resultados en rendimiento: $3,42 \mathrm{Kg}$. de peso seco de la primera más la segunda cosecha y 6,33 TM/. Ha rendimiento comercial. Conclusión: El mayor rendimiento comercial del aji paprika se obtuvo a $20 \mathrm{~cm}$ de distanciamiento entre plantas, en relación a los otros parámetros evaluados no fueron afectados por las densidades de sembrios.
\end{abstract}

Palabras clave: Ají paprika, densidad de siembra, rendimiento comercial y calidad.

\section{ABSTRACT}

Objective: To evaluate the planting densities with respect to the yield of the paprika pepper (Capsicum annuum L.), in the Bella variety, to determine which distancing obtained the highest yield and quality in the La Florida Sector - Barranca Province. Material and Methods: The completely randomized block design was used, consisting of four treatments and four blocks and for the operation of the data the analysis of variance and the Duncan test. They were evaluated from transplant to post harvest, in the distances $(T)$ of $T 1=20 \mathrm{~cm}, \mathrm{~T} 2=25 \mathrm{~cm}, \mathrm{~T} 3=30 \mathrm{~cm}$ and $\mathrm{T} 4=35 \mathrm{~cm}$ at $90 \mathrm{~cm}$ between rows. Results: The treatment T4=35 cm. (greater distancing), excelled in the following parameters as: $97.25 \%$ yield percentage, $75.25 \mathrm{~cm}$. plant height, 15 fruits per plant; 33 flowers per plant; 10 ripe fruits per plant; $30.51 \mathrm{~g}$. of fresh fruits; $6.99 \mathrm{~g}$ of dried fruit per plant; $14.76 \mathrm{~cm}$ fruit length; $3.46 \mathrm{~cm}$ in diameter, $0.50 \mathrm{~cm}$. Although the T4 showed good characteristics; The T1 $=20 \mathrm{~cm}$. (less distancing), good results were also obtained in yield: $3.42 \mathrm{~kg}$ dry weight of the first plus the second harvest and $6.33 \mathrm{MT} /$. It has commercial performance. Conclusion: The highest commercial yield of aji paprika was obtained at a distance of $20 \mathrm{~cm}$ between plants, in relation to the other parameters evaluated, they were not affected by sowing densities.

Keywords: Chili pepper, planting density, commercial performance and quality.

\section{INTRODUCCIÓN}

Durante muchos años el agricultor ha sembrado en distanciamientos inadecuados, por lo que no ha tenido buen rendimiento y calidad en el ají paprika, por lo que es necesario conocer que espacio entre planta es favorable. Esto se relaciona con lo indicado por Vilmorín(1977) que recomienda: "Para el plantín dulce tipo ají páprika variedad Sonora, trasplantar en surcos separados de 0.9 $\mathrm{m}$. - $1 \mathrm{~m}$. y de $18 \mathrm{~cm}$. - $30 \mathrm{~cm}$., entre plantas" (p.314). Mientras que el INIA de México indica para el mismo pimiento siembras en surco separado de $1 \mathrm{~m}$. $-1.5 \mathrm{~m}$., y entre plantas 20 - $25 \mathrm{~cm}$." (p.314).

Al respecto el Grupo Poblete (2000), también indica que: "En el sistema de riego tradicional por gravedad el distanciamiento debe ser 0,90 a $1 \mathrm{~m}$. Entre surco y entre planta de 0,20 a 0,25 m; los cuales hacen una densidad de 55555 a 44444 plantas por ha. En el sistema de riego tecnificado o presurizado el espacio entre surco puede variar de 1,20 a 1,40 m. y la distancia entre plantas de $0,15 \mathrm{~m}$. a $0.20 \mathrm{~m}$. en siembra melliza, los cuales hacen una población de 111111 a 71429 plantas por ha." (p.23).

La mayoría de los productores están usando densidades de siembra probadas con buenos resultados, al respecto Zegarra (2000), afirma que: "La densidad de siembra más empleado por los agricultores oscila en 66666 y 62 500 plantas por hectárea (esta densidad se realiza entre surco de $0,75-0,80 \mathrm{~m}$. y entre planta $0.20 \mathrm{~m}$.)" (p.48).
Respecto a las referencias donde se evalúa los rendimientos según las densidades de siembra, se tiene que Lozada (1990) señala que: "Ios rendimientos más altos del pimiento dulce fueron obtenidos en densidades de siembra de 60000 plantas /ha, resultado de la siembra directa en tres bolillos, con 2 golpes entre plantas de 33 $\mathrm{cm}$. y separación de $1 \mathrm{~m}$ entre cintas de exudación. El cultivar "Bell Boy" consiguió el rendimiento más alto en dicha densidad de siembra en comparación con el Bing Star" (p.58).

Cabe mencionar que este trabajo tiene como propósito las evaluaciones de los distanciamientos de ají páprika variedad Bella establecidos en la investigación, lo cual se determinará que distanciamiento produce mayor rendimiento y calidad de frutos. $Y$ de esta manera precisar que densidad es adecuada para la rentabilidad.

Por tal razón el objetivo central es evaluar las cuatro densidades de ají páprika (Capsicum annuum L.), variedad. Bella, para la obtención de mayor rendimiento y calidad, en el sector La Florida - Provincia de Barranca. 


\section{MATERIALES Y MÉTODOS}

La investigación es de tipo aplicada; ya que los resultados se obtendrán mediante evaluaciones continuas y servirá como recomendación para obtener mayor rendimiento y calidad en el cultivo de aji Paprika.

\section{Análisis de varianza:}

La operación estadísticas que se aplicó fue el análisis de varianza (ANVA), con la prueba de $\mathrm{F}$ de homogeneidad al $5 \%$ y $1 \%$, que determinó que tratamientos son significativos o no, esto se fundamenta con Morales (2012) que afirma: "El análisis de varianza se utiliza para verificar si hay diferencias estadísticamente significativas entre medias cuando tenemos más de dos muestras o grupos en el mismo planteamiento" (p.2).

Tabla 1

Componentes de la Tabla de análisis de varianza

\begin{tabular}{lcclc}
\hline $\begin{array}{c}\text { Fuente de } \\
\text { variación }\end{array}$ & $\begin{array}{c}\text { Suma de los } \\
\text { cuadrados }\end{array}$ & $\begin{array}{c}\text { Grados de } \\
\text { libertad }\end{array}$ & Media cuadrática & F \\
\hline Tratamientos & SST & $\mathrm{k} 1$ & $\mathrm{SST} /(\mathrm{k} \mathrm{1})=\mathrm{MST}$ & $\mathrm{MST} / \mathrm{MSE}$ \\
Bloques & $\mathrm{SSB}$ & $\mathrm{b} 1$ & $\mathrm{SSB} /(\mathrm{b} 1)=\mathrm{MSB}$ & $\mathrm{MSB} / \mathrm{MSE}$ \\
Error & $\mathrm{SSE}$ & $\mathrm{k}$ 1)(b 1) & $\mathrm{SSE} /(\mathrm{k} \mathrm{1})(\mathrm{b} \mathrm{1)}=\mathrm{MSE}$ & \\
total & $\mathrm{SS} \mathrm{Total}$ & $\mathrm{n} \mathrm{1}$ & & \\
\hline
\end{tabular}

Fuente: Lind, D.et. al (2012), "Estadística Aplicada a los Negocios y la Economía".

\section{Prueba de Duncan:}

Se operó los datos con la prueba comparativa de Duncan, esto determinó si hubo homogeneidad de los resultados de los tratamientos, pues según Condo y Pazmiño (2015) sostienen que: "Es una prueba más estricta que la DMS (Diferencia Mínima Significativa), de esta manera, permite comparar todas las medias entre sí, sin restricciones"(p.91).

\section{Tratamientos}

El trabajo de investigación se estudió con un solo factor que es las densidades de siembra de ají paprika Variedad Bella en lo cual comprende el distanciamiento entre plantas. (Tabla 2).

Tabla 2

Densidad de la siembra de Ají Paprika variedad Bella.

\begin{tabular}{ccc}
\hline Tratamiento & Espacio entre surco y plantas & Densidades / ha \\
\hline $\mathrm{T}_{1}$ & $0,90 \mathrm{~m} \times 0,20 \mathrm{~m}$. (2 plantas por golpe) & 111111 plantas \\
$\mathrm{T}_{2}$ & $0,90 \mathrm{~m} \times 0,25 \mathrm{~m}$. (2 plantas por golpe) & 88888 plantas \\
$\mathrm{T}_{3}$ & $0,90 \mathrm{~m} \times 0,30 \mathrm{~m}$. (2 plantas por golpe) & 74074 plantas \\
$\mathrm{T}_{4}$ & $0,90 \mathrm{~m} \times 0,35 \mathrm{~m}$. (2 plantas por golpe) & 63492 plantas \\
\hline
\end{tabular}

Fuente de investigación: El autor (2016).

\section{RESULTADOS}

\section{Altura de planta}

De acuerdo a los resultados del análisis de varianza, que se indica en la tabla, se determinó que no hubo diferencias estadísticas entre tratamientos y bloques, lo cual significa que no influyeron los distanciamientos en la altura de planta. Asimismo se observa el coeficiente de variación es de $4,5 \%$, lo que se interpreta que hay una ligera variación. ( Figura 1 y Tabla 3)

\section{Cantidad de frutos}

En la Tabla 3, se aprecia que no son significativos, siendo todos los tratamientos estadísticamente homogéneos. Por lo tanto no hubo efecto por el distanciamiento entre plantas a distancia de surco de $0,75 \mathrm{~m}$. Asimismo se detalla que la mayor cantidad de frutos los obtiene el $\mathrm{T}_{4}$ con 15,25 unidades.

\section{Peso de fruto fresco por planta}

Desarrollado el análisis estadístico se determinó que hubo diferencias estadísticas entre tratamientos, es decir es hubo efecto de la densidad de siembra. También se observa que sobresalió el $\mathrm{T}_{4}$ con $422,80 \mathrm{~g}$ por planta operando con la prueba de Duncan al $5 \%$ ( Tabla 3)

\section{Peso de fruto seco por planta}

Relacionado al peso seco por planta se determinó mediante el análisis de varianza que fue significativo; es decir hubo efecto de densidad de siembra. Cabe afirmar que la operación de la prueba de Duncan estableció que el $\mathrm{T}_{4}$ con 99,45 (Ver Tabla 3)

\section{Rendimiento comercial}

Con respecto al rendimiento comercial, se determinó por medio del análisis de varianza que no hubo diferencias estadísticas, es decir no hubo efecto de distanciamiento y con la prueba de Duncan resaltó que el $T_{1}$ obtuvo 6,33 $\mathrm{tn} /$ ha. (Tabla 3)

\section{Peso de un fruto seco}

En cuanto a la evaluación de calidad de peso de un fruto seco que se indica en la tabla 4 , se determinó por medio del análisis de varianza que no hubo diferencias estadísticas entre tratamiento, por lo que no influyó el distanciamiento. Cabe mencionar que la operación de la Prueba de Duncan destaca el $\mathrm{T}_{4}$ con 6,99 g diferenciándose de los demás. 


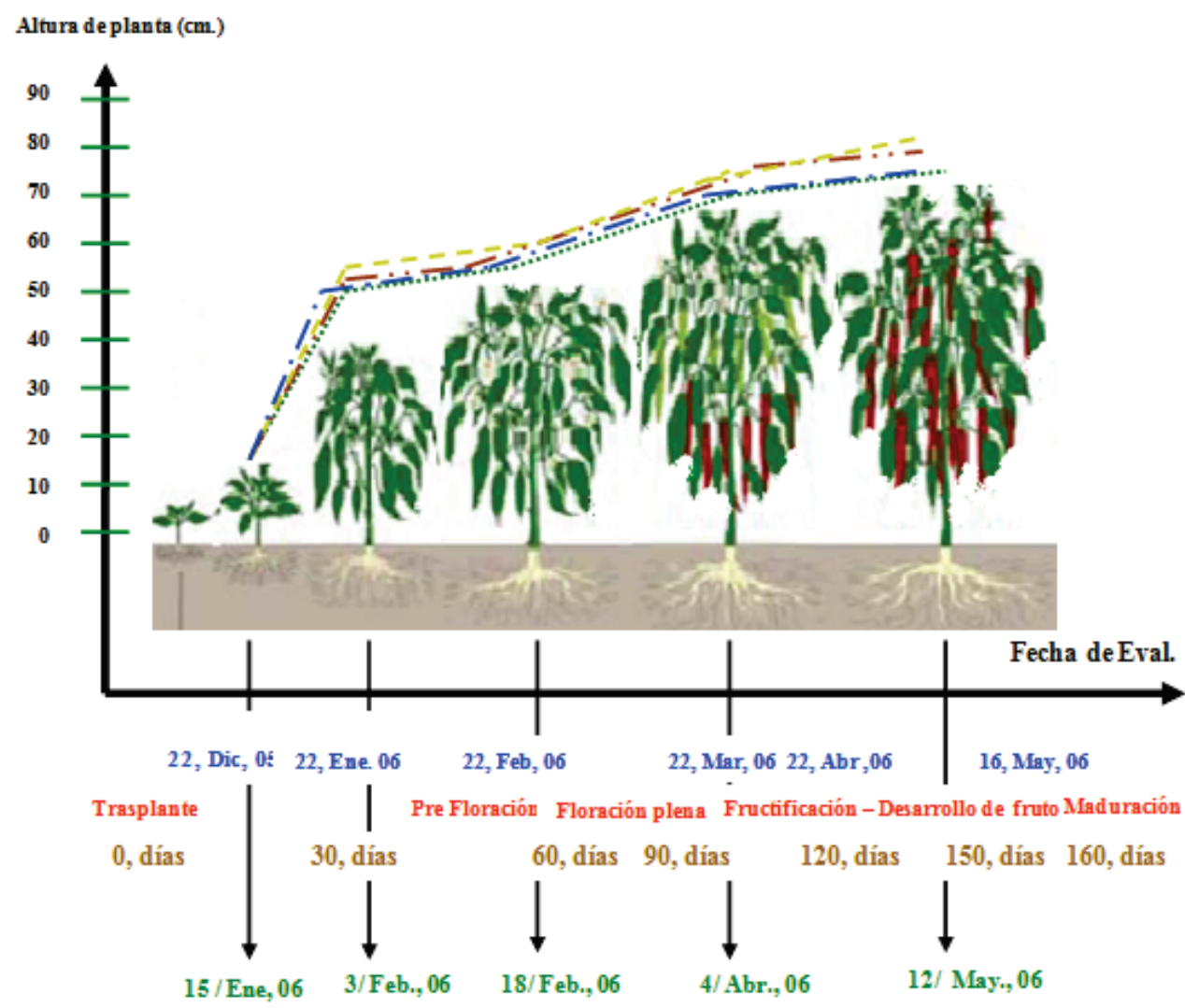

Figura 1. Altura de planta en función a los tratamientos

Promedios de altura de plantas según las fechas

\begin{tabular}{ccccc} 
& \multicolumn{4}{c}{ Tratamiento $(\mathrm{cm}$.) } \\
\cline { 2 - 5 } F. evaluación & $\mathbf{T}_{\mathbf{1}}$ & $\mathbf{T}_{\mathbf{2}}$ & $\mathbf{T}_{\mathbf{3}}$ & $\mathbf{T}_{\mathbf{4}}$ \\
\hline 15/01/06 & 16,07 & 15,45 & 16,45 & 15,50 \\
$03 / 02 / 06$ & 48,82 & 48,90 & 51,10 & 52,12 \\
18/02/06 & 52,47 & 53,22 & 52,22 & 54,95 \\
$04 / 04 / 06$ & 67,85 & 70,03 & 71.55 & 71,77 \\
$12 / 05 / 06$ & 70,27 & 71,15 & 73,20 & 75,72 \\
\hline
\end{tabular}

Tabla 3

Resultados de las características físicas en campo de aji páprika variedad bella

\begin{tabular}{|c|c|c|c|c|c|c|}
\hline Tratamiento & $\begin{array}{l}\text { Distancia } \\
\text { entre plantas } \\
\quad \text { (cm.) }\end{array}$ & $\begin{array}{l}\text { Altura } \\
\text { de planta } \\
(\mathrm{cm})\end{array}$ & $\begin{array}{l}\text { Cantidad de } \\
\text { fruto ( } \mathrm{g} / \text { planta) }\end{array}$ & $\begin{array}{l}\text { Peso de fruto } \\
\text { fresco (g./ } \\
\text { planta) }\end{array}$ & $\begin{array}{l}\text { Peso de fruto } \\
\text { seco (g./ } \\
\text { planta)) }\end{array}$ & $\begin{array}{l}\text { Rendimiento } \\
\text { comercial } \\
\text { (tn/ha) }\end{array}$ \\
\hline $\mathrm{T}_{4}$ & $0,35 \mathrm{~m}$ & $75,25 a$ & $15,25 \mathrm{a}$ & $422,80 \mathrm{a}$ & $99,45 a$ & $5,0130 \mathrm{~b}$ \\
\hline $\mathrm{T}_{3}$ & $0,30 \mathrm{~m}$ & $73,20 \mathrm{a}$ & $12,50 \mathrm{a}$ & $375,68 a b$ & $88,38 a b$ & $5,2281 b$ \\
\hline $\mathrm{T}_{2}$ & $0,25 \mathrm{~m}$ & $71,15 a$ & $12,00 \mathrm{a}$ & $340,06 \mathrm{~b}$ & $80,00 \mathrm{~b}$ & $5,6355 a b$ \\
\hline $\mathrm{T}_{1}$ & $0,20 \mathrm{~m}$ & $70,27 a$ & 12,25 a & $329,22 \mathrm{~b}$ & $77,465 \mathrm{~b}$ & $6,3306 \mathrm{ab}$ \\
\hline \multicolumn{2}{|c|}{ Coeficiente de variación } & 4,5 & 16,61 & 8,50 & 8,10 & 11,7 \\
\hline \multicolumn{2}{|l|}{ Significación } & ** & ** & ${ }^{*}$ & * & $* *$ \\
\hline
\end{tabular}

Nota. Promedio con la misma letra son estadísticamente homogéneos $\left.{ }^{*}\right)$ Significativo y $\left({ }^{* *}\right)$ no significativo Fuente: El autor (2016) 


\section{Tabla 4}

Resultados de las características de calidad de ají páprika.

\begin{tabular}{ccccccc}
\hline Tratamiento & $\begin{array}{c}\text { Distancia } \\
\text { entre plantas } \\
(\mathrm{cm})\end{array}$ & $\begin{array}{c}\text { Peso de un } \\
\text { fruto por } \\
\text { planta }(\mathrm{g})\end{array}$ & $\begin{array}{c}\text { Peso de un } \\
\text { fruto seco } \\
(\mathrm{g})\end{array}$ & $\begin{array}{c}\text { Longitud de un } \\
\text { fruto }(\mathrm{cm})\end{array}$ & $\begin{array}{c}\text { Diámetro } \\
\text { de un fruto } \\
(\mathrm{cm})\end{array}$ & $\begin{array}{c}\text { Grosor de } \\
\text { pericarpio } \\
(\mathrm{cm})\end{array}$ \\
\hline $\mathrm{T}_{4}$ & $0,35 \mathrm{~m}$ & $30,51 \mathrm{a}$ & $6,99 \mathrm{a}$ & $14,76 \mathrm{a}$ & $3,46 \mathrm{a}$ & $0,50 \mathrm{a}$ \\
$\mathrm{T}_{3}$ & $0,30 \mathrm{~m}$ & $29,04 \mathrm{a}$ & $6,78 \mathrm{a}$ & $14,72 \mathrm{a}$ & $3,37 \mathrm{a}$ & $0,49 \mathrm{a} a$ \\
$\mathrm{~T}_{2}$ & $0,25 \mathrm{~m}$ & $28,17 \mathrm{a}$ & $6,72 \mathrm{a}$ & $14,58 \mathrm{a}$ & $3,35 \mathrm{a}$ & $0,49 \mathrm{a}$ \\
$\mathrm{T}_{1}$ & $0,20 \mathrm{~m}$ & $27,92 \mathrm{a}$ & $6,56 \mathrm{a}$ & $14,54 \mathrm{a}$ & $3,33 \mathrm{a}$ & $0,48 \mathrm{a}$ \\
\hline \multicolumn{2}{l}{ Coeficiente de variación } & 5,25 & 4,96 & 4,96 & 2,21 & 2,04 \\
\hline \multicolumn{2}{l}{ Significación } & & $* *$ & $* *$ & & $* *$ \\
\hline
\end{tabular}

Nota. Promedio con la misma letra son estadísticamente homogéneos

${ }^{*}$ ) Significativo y $\left({ }^{* *}\right)$ no significativo

Fuente: El autor (2016)

\section{Longitud de un fruto}

Seguido a la evaluación de longitud de un fruto que se detalla los resultados en la tabla 4, que precisó por medio del análisis de varianza que no hubo efecto en los distanciamientos; es decir no son significativos De la misma manera se aprecia el resultado de la prueba de Duncan que resaltó el $\mathrm{T}_{4}$ con $14,76 \mathrm{~cm}$ de longitud.

\section{Diámetro de un fruto}

Respecto al diámetro de un fruto que se expone en la tabla 4, se determinó que no son significativos; es decir no influyeron los distanciamientos. Asimismo se aprecia que el resultado de la prueba de Duncan destacó que el $\mathrm{T}_{4}$ obtuvo $3,46 \mathrm{~cm}$.

\section{DISCUSIÓN}

El mayor número de frutos por planta se obtuvo en el mayor distanciamiento entre plantas presentado buen vigor y desarrollarse libremente en comparación al de menor distanciamiento que presentó dificultad por la competencia nutriente y la formación de un microclima por sus ramas ocasionando la caída de algunos frutos. Estos resultados fueron corroborados por Núñez, (2000) que trabajó con dos cultivares de pimiento páprika en tres densidades de siembra en la Zona de Yarada (Tacna), obteniendo promedios en número de frutos por planta, para el cultivar de Papri king 25,33; 15,57; 42 papri queen 18,$67 ; 20,33 ; 28,67$ en los distanciamientos de 20,30 y $40 \mathrm{~cm}$. entre plantas.

En cuanto al peso de fruto fresco por planta, se determinó que el $\mathrm{T}_{4}$ obtuvo mayor peso con $422,8 \mathrm{~g} /$ planta, esto se debe a que la competencia nutricional entre plantas no fue significativa, mayor ventilación y eficiencia en el control de plagas, lo cual fue favorable en el mayor peso. Esto se fundamenta con Núñez (2000) obtuvo: "peso promedios en gramo de fruto fresco por planta, en el cultivar de papri King con: 32,50; 35,83 y 35 g., para los distanciamiento de 20.30 y $40 \mathrm{~cm}$, determinando que a mayor espacio entre planta se obtendrá mayor peso por fruto fresco por planta" (p.33).

De acuerdo a los resultados de la Tabla 3, se aprecia que el mayor rendimiento comercial se obtuvo con el $T_{1}$ obtuvo 6,33 tn/ ha, diferenciándose de lo demás tratamientos; por lo que influyó la mayor cantidad de plantas por ha; sin embargo no sobresalió en calidad, esto se puede contrastar con Paredes (2017), expone "El mayor peso de frutos se obtuvo con el tratamiento 3 $(0,9 \mathrm{~m} \times 0,7 \mathrm{~m})$ (15 873 plantas por hectárea) que alcanzó $2556,25 \mathrm{~kg}$.ha-1, así mismo la mayor utilidad se obtuvo con el mismo tratamiento que registró una relación beneficio costo de 1,17; en comparación al distanciamiento $T_{4}$ con $(0,8 \times 0,9) 13889$ plantas por hectárea.

Respecto a la evaluación en calidad, se determinó que el mayor peso de un fruto seco se obtuvo en el $\mathrm{T}_{4}$ con $6,99 \mathrm{~g}$ diferenciándose de los demás. Este resultado se debe que a mayor distanciamiento obtuvo mayor peso de un fruto seco, ya sea por la menor competencia nutricional entre planta, mayor ventilación que beneficia en el control de plagas y otros. Dicha afirmación se relaciona con Núñez (2000) que tuvo resultados en el cultivar de papri King con 6,$5 ; 6.7$ y 6,8 g., para los distanciamiento de 20, 30 y $40 \mathrm{~cm}$. entre planta.

Seguido a la evaluación de longitud de un fruto, se aprecia en la Tabla 4 que el mayor tamaño se obtuvo en el $\mathrm{T}_{4}$ con $14,76 \mathrm{~cm}$, lo que se interpreta que a este distanciamiento se favoreció el desarrollo de futo; ya que a mayor distanciamiento mayor desarrollo del fruto, menor competencia nutricional y mayor ventilación. Este análisis se fundamenta con Lindao (2002) quien realizó, una investigación de "Cuatro densidades de siembra en el cultivo de pimiento páprika (Capsicum annuum L.) en el Valle de Chancay- Huaral", donde obtuvo resultados de 12,$9 ; 12,78 ; 12,97$ y $13,06 \mathrm{~cm}$. de longitud de fruto, para los distanciamientos de 20,30, 40 y $50 \mathrm{~cm}$. entre plantas afirma que no se encontró diferencias significativas" (p.36).

Concerniente al diámetro de un fruto, se preció que el mayor ancho se obtuvo en el $\mathrm{T}_{4}$ con $3,46 \mathrm{~cm}$, lo cual beneficia en la calidad ya sea por sus condiciones de mayor presencia, tamaño y por su volumen. Estas características benefician al agricultor en su comercialización, puesto que es muy aceptado en los mercados de acopio. Analizado este resultado se contrasta con la investigación de Lindao (2002) que obtuvo promedios en el diámetro de fruto $(\mathrm{cm}$.) en la variedad papri King, siendo estos 1,$18 ; 1,97 ; 1,83$ y 2,18 $\mathrm{cm}$., para los distanciamientos de $20,30,40$ y $50 \mathrm{~cm}$. entre planta. Los espacios entre planta no influenciaron significativamente en el diámetro de fruto, pero un ligero aumento en diámetro a mayor separación entre planta" (p.36). 


\section{CONCLUSIONES}

El mayor rendimiento comercial de la variedad Bella se obtuvo a $20 \mathrm{~cm}$. de distanciamiento entre plantas, obteniéndose en peso seco 6,330 TM / Ha. superando a las otras densidades en estudio. El más bajo fue de 35 cm. entre plantas 5,013 TM. /ha. peso seco.

Se obtuvo un mayor número de frutos, además en peso fresco y seco por planta en el distanciamiento de $35 \mathrm{~cm}$. entre planta, sin embargo esto no trascendió para el rendimiento total por tratamiento.

Se presentó un mayor porcentaje de prendimiento, vigor de planta y buena presencia de frutos en los tratamientos de 30 y $35 \mathrm{~cm}$. entre plantas, esto debido al mayor distanciamientos, mayor horas luz y menor competencia de nutrientes.

En cuanto a las demás evaluaciones se observó que el porcentaje de prendimiento, altura de planta $(\mathrm{cm}$.), días de inicio de floración por tratamiento, número de flores por planta, longitud de diámetro $(\mathrm{cm}$.) y grosor del pericarpio $(\mathrm{cm}$.), no fueron afectados por las densidades, de siembra, es decir se mantuvo un patrón semejante.

\section{REFERENCIAS BIBLIOGRÁFICAS}

Condo, L. y Pazmiño, J. (2015), “Diseño experimental en el desarrollo del conocimiento científico de las ciencias agropecuarias". Tomo $\mathrm{N}^{\circ} 1$. Escuela Superior Politécnica de Chimborazo e instituto de Investigaciones. Ecuador. Página 91

Grupo Poblete, (2000) "El cultivo de páprika productora de semillas S.A". Chosica- Lima. Ficha técnica 23 pp.- 30 pp.

Lind, D.; Marchal, W. y Wathen, S. (2012), "Estadística Aplicada a los Negocios y la Economía”. Coastal Carolina University and University of Toledo EE.UU. Traducción de: Jorge Yescas y Javier León Cárdenas- México. Página 432.
Lindao, C. (2002), "Evaluación de cuatro densidades de siembra de ají páprika (Capsicum annuum L.), en el valle de Chancay- Huaral". Tesis de la Universidad de Tumbes-Perú 36-37 pp.

Lozada. A., (1990). Efecto de cinco densidades de siembra directa en la producción de cinco híbridos de pimiento dulce (Capsicum annuum L.) bajo riego de alta frecuencia (micro exudación) tesis Universidad Nacional Agraria La Molina. Lima-Perú $58 \mathrm{pp}$.

Morales, P. (2012), "Introducción al Análisis de Varianza”. Universidad Pontificia Comillas. España página 2.

Núñez, H. (2000). Ensayo de tres densidades de siembra en dos cultivares de pimiento páprika (Capsicum annuum L.) en Yarada - Tacna. Tesis de la Universidad Nacional Agraria La Molina - Lima Perú 33-51 pp.

Paredes, J. (2017), "Evaluación de cuatro densidades de siembra en el rendimiento del cultivo de ají charapita (Capsicum frutescens L.) en el sector Almendras, provincia de Tocache", Tesis para optar el título profesional de Ingeniero Agrónomo. Universidad Nacional de San Martín - Tarapoto. Página 23 y 42

Vilmorín, F., (1977). Cultivo de pimiento dulce tipo Bell. Editorial Diana México 314 pp.

Zegarra, J., (2000). Adopción del cultivo de pimiento páprika (Capsicum annuum L.), por los agricultores del Valle de Vitor Arequipa. Tesis Universidad Nacional Agraria La Molina, Lima-Perú 48 pp. 